\title{
3 and 12 lead electrocardiogram interpretation by computer A comparison on 1093 patients
}

\author{
Peter W. Macfarlane, A. R. Lorimer, and T. D. V. Lawrie \\ From the University Department of Medical Cardiology, \\ Royal Infirmary, Glasgow C.4
}

A comparative study of computer interpretations of 3 and 12 lead electrocardiograms from 1093 patients is reported. The conclusion reached is that 3 lead electrocardiography is clinically as acceptable as 12 lead electrocardiography with respect to computer assisted interpretation.

The use of automated methods for electrocardiogram interpretation is not new. Procedures have been developed for the analysis of 12 lead electrocardiograms (Caceres et al., 1962; Bonner and Schwetman, 1968) and for the interpretation of 3 lead electrocardiograms (Stallmann and Pipberger, I96I; Pipberger and Stallmann, 1964; Smith and Hyde, 1969). More recently a technique has been developed (Macfarlane, 197I) for use with either lead system, when leads are recorded in groups of 3 simultaneously. There have, however, been few comprehensive comparisons of 3 and 12 lead electrocardiogram interpretations by computer or few studies of computer interpreted orthogonal lead electrocardiograms with lead systems other than that of Frank (1956). A modification to the axial lead system of McFee and Parungao (I96I) has been described (Macfarlane, 1969) which effectively equalized the lead strengths previously shown to be unequal (Brody and Arzbaecher, 1964). This modification was designed to make the axial lead system approximate to the ideal orthogonal lead system, with mutually perpendicular lead vectors of equal magnitude. While this modified axial lead system was theoretically acceptable it remained to be shown whether or not it was clinically as useful as the conventional 12 lead system with the assistance of computer interpretation.

In the technique of 12 lead electrocardiography 12 projections of the cardiac electrical activity (Fig. I) in different directions are recorded sequentially. In 3 lead electrocardiography only 3 projections, leads $\mathrm{X}, \mathrm{Y}, \mathrm{Z}$, are Received I September 1970. simultaneously recorded in mutually perpendicular directions (Fig. I). These leads can be combined in groups of two for the formation of the vectorcardiogram (Fig. 2) which must contain the same information as its equivalent 3 scalar lead display. The techniques used for computer analysis of the 3 lead electrocardiogram require only the recording of the $\mathrm{X}, \mathrm{Y}$, and $\mathrm{Z}$ leads, since the computer programme can use these data to calculate the vectorcardiogram from simple mathematical formulae.

Controversy over the relative merits of 3 and 12 lead electrocardiography has continued over the past decade. Pipberger et al. (196r), using visual interpretation methods, claimed that the two lead systems were equivalent. Borun, Chapman, and Massey (1966) compared computer measurements of Frank system 3 lead electrocardiograms with 12 lead abnormalities determined visually. Their results were equivocal and further comparisons were suggested. Simonson et al. (I966) showed that the I2 lead electrocardiogram was more accurately interpreted by cardiologists than the 3 lead, though this co-operative study was handicapped by the unfamiliarity of some observers with the 3 lead system used. These authors found that all the information contained in the 3 lead electrocardiogram had not been used by the cardiologists and it was concluded that further quantitative analyses using computer methods were desirable. Spach et al. (1966) then emphasized the value of incorporating buffer amplifiers between the patient electrode and the electrocardiogram amplifier, while Berson and Pipberger (1967) showed that without such circuitry potential 


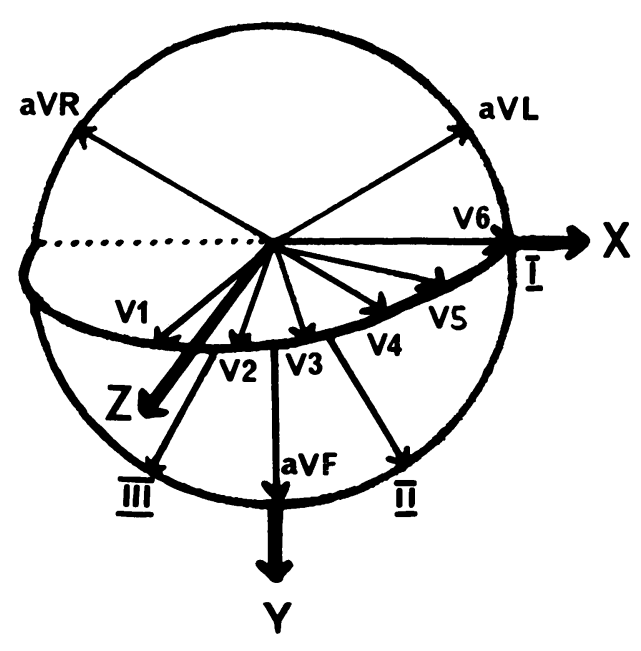

FIG. I An illustration of electrocardiogram lead directions. The leads $I, I I, I I I, a V R$, $a V L, a V F, X, Y$ are in the frontal plane and leads $V I-V 6$ are in the transverse plane.

measurements could vary by as much as 50 per cent in the 3 lead electrocardiogram during 15 minutes after the application of electrodes. These findings suggested that further comparisons of both lead systems be carried out with more suitably designed equipment than previously used.

The study reported in this paper was undertaken to determine which of the two lead systems would be more suitable for routine electrocardiogram interpretation by computer.

\section{Methods}

There were ro93 subjects in this study. Each was assessed on the basis of physical examination, I2 lead electrocardiogram, and $x$-ray of chest. Patients with rheumatic valvular disease had haemodynamic and angiographic studies carried out at cardiac catheterization. Patients with myocardial infarction had serum enzyme changes measured routinely. The clinical classification of the rog3 patients is shown in Table $\mathrm{I}$.

Each patient had a 12 lead electrocardiogram and a modified axial system 3 lead electrocardiogram tape recorded either in the ward or in the laboratory. Hewlett Packard 2700 I2 lead electrocardiogram amplifiers and a $1520 \mathrm{~A}$ Vector Programmer, which incorporated buffer amplifiers, were used. Initially each 12 lead electrocardiogram recorded in groups of 3 leads simultaneously, and each 3 lead cardiogram was replayed to a PDP8 computer for analogue to digital conversion. Data were subsequently analysed on a $\mathrm{KDF}_{9}$ computer by methods to be outlined elsewhere (Macfarlane, 197I). The result of the analysis was a set of all $P, Q, R, S$, and $T$ wave amplitude and duration measurements, selected vector magnitudes, and orientations such as 0.01 , 0.02 sec., etc., after QRS onset and time normalized data, that is measurements made at $\mathrm{r} / 8,2 / 8$, etc., of the QRS complex and the ST-T segment. Each 12 lead electrocardiogram was interpreted by a further computer programme which incorporated data from established studies such as those of Simonson (196I), New York Heart Association (1964), Sokolow and Lyon (1949a, b),

FIG. 2 The leads $X, Y, Z$ of a 3 lead electrocardiogram which are also combined in pairs to form the frontal $(X, Y)$, right sagittal $(Y, Z)$, and transverse $(X, Z)$ loops of the vectorcardiogram. The timing dashes are marked at intervals of $2.5 \mathrm{msec} . F=$ frontal. $R S=$ right sagittal. $T=$ transverse.
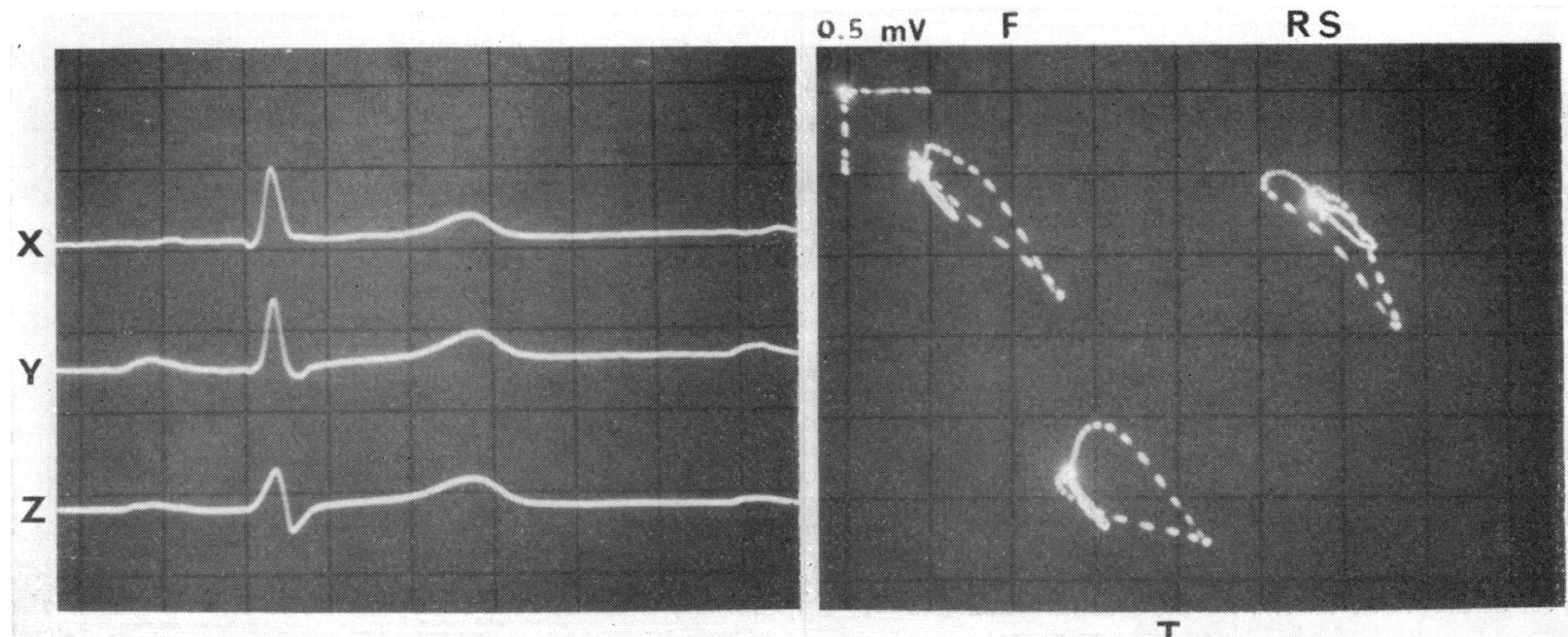
TABLE I Clinical classification of the I093 patients, whose ages ranged from 4 to 80 years

\begin{tabular}{lr}
\hline Normal & 221 \\
Myocardial infarction & I I4 \\
Myocardial ischaemia & 62 \\
Hypertension & 91 \\
Congenital heart disease & 33 \\
Acquired heart disease & I42 \\
Respiratory disease & 58 \\
Alimentary disease & 76 \\
Endocrine disease & 85 \\
Renal disease & 44 \\
Blood disease & 43 \\
Neurological disease & 66 \\
Pulmonary vascular disease & 21 \\
Miscellaneous & 37 \\
\hline
\end{tabular}

TABLE 21093 I2 lead electrocardiogram interpretations as agreed by the panel of cardiologists

\begin{tabular}{cr} 
Clinically normal group A & 205 \\
Clinically normal group B & 125 \\
Other & 148 \\
Myocardial infarction: Anteroseptal & 55 \\
Inferior & 40 \\
Other & 14 \\
Left ventricular hypertrophy & 141 \\
Right ventricular hypertrophy & 50 \\
Biventricular hypertrophy & 13 \\
Myocardial ischaemia: Inferior & 30 \\
Lateral & 42 \\
Anteroseptal & 24 \\
Inferolateral & 40 \\
Conduction defects & 39 \\
Miscellaneous: P wave abnormalities & 28 \\
Minor ST abnormalities & 28 \\
Low voltage & 12 \\
Tall T waves & 33 \\
Axis deviation & 26 \\
\hline & 1093
\end{tabular}

etc. A panel of cardiologists also interpreted each 12 lead electrocardiogram on the basis of the same criteria. It was thus possible to check for computer or panel interpretation variations. In the event of a discrepancy the electrocardiogram was reviewed by the panel, who made the final decision on its interpretation. Table 2 shows the interpretations of the 1093 I2 lead tracings.

A study was made of 265 patients with a normal I2 lead electrocardiogram and a normal cardiovascular system in order to obtain normal ranges for the modified axial system 3 lead electrocardiogram (Macfarlane, Lorimer, and Lawrie, I97I). Similar statistical analyses involving means and 96 per cent ranges were made for each group of 3 lead electrocardiograms which corresponded with each abnormal i2 lead group. From these results a set of diagnostic criteria for the modified axial system was established. These were incorporated into a third computer programme which used the results of the wave measurement programme to produce a 3 lead electrocardiogram interpretation. An example of corresponding 3 and 12 lead computer interpretations is shown in Fig. 3. For both lead systems the method of interpretation was to check wave amplitudes, etc., against normal limits as opposed to a multidimensional statistical approach.

\section{Results}

The first comparison concerned the incidence of false positive and false negative computer interpretations.

False positive computer interpretations The incidence of false positive computer interpretations was obtained from a study of a subgroup of 205 subjects who were clinically normal. The group consisted of student volunteers and others admitted to hospital for minor surgical procedures. All had a normal 12 lead electrocardiogram as determined by the panel of cardiologists. Since each patient was clinically normal with a normal in lead electrocardiogram, each corresponding 3 lead electrocardiogram and its computer interpretation was expected to be normal.

The more important false positive computer interpretations are shown in Table 3. There is no significant difference between either lead system though a slightly higher prevalence of 3 lead electrocardiogram false positive interpretations might have been expected. This follows from the fact that, since all of the 205 patients belonged to the group of 265 from whom the 96 per cent normal limits were obtained, false positive results could arise from normal patients whose 3 lead electrocardiogram parameters were outside the $96 \%$ normal ranges, even though diagnostic criteria did not always use these limits as the boundary between normal and abnormal.

TABLE 3 False positive computer interpretations as determined from 205 normal patients

\begin{tabular}{lcc}
\hline Computer interpretation & \multicolumn{2}{l}{ Lead system } \\
\cline { 2 - 3 } & I2 lead & 3 lead \\
\hline Left ventricular hypertrophy & 6 & 7 \\
Right ventricular hypertrophy & 5 & 9 \\
Myocardial infarction & 5 & 3 \\
Myocardial ischaemia & 7 & 9 \\
ST elevation & 8 & 10 \\
Tall T waves & 12 & 7 \\
Conduction defects & I I & 6 \\
Low voltage & I & 3 \\
\hline & 55 & 54 \\
\hline
\end{tabular}


3 LEAD ECG INTERPRETATION

PATIENT NUMBER 937

SINUS BRADYCARDIA 49 PER MINUTE

PR INTERVAL NORMAL

ABNORMALLY DEEP S WAVE IN ANTEROSEPTAL LEAD

APPEARANCES ARE CONSISTENT WITH LEFT VENTRICULAR HYPERTROPHY

TALL T WAVES POSSIBLY DUE TO LV HYPERTROPHY

NIL ELSE OF NOTE

I2 LEAD ECG INTERPRETATION

PATIENT NUMBER 937

SINUS BRADYCARDIA 49 PER MINUTE

PR INTERVAL NORMAL

ABNORMALLY HIGH PRAECORDIAL QRS VOLTAGES FOR A PATIENT OF THIS AGE

APPEARANCES ARE CONSISTENT WITH LEFT VENTRICULAR HYPERTROPHY

TALL T WAVES POSSIBLY DUE TO LV HYPERTROPHY

NIL ELSE OF NOTE

FIG. 3 An example of corresponding 3 and 12 lead computer interpretations, copied from the computer printout for clarity.

False negative computer interpretations The evaluation of false negative interpretations was more difficult because of lack of necropsy data. A group of 419 patients who were regarded by the panel as having frankly abnormal I2 lead tracings were studied. For the purposes of this test it was assumed that the 3 lead electrocardiogram should at least show the same abnormalities as the corresponding abnormal i2 lead electrocardiogram. Table 4 shows the more impor-

TABLE 4 False negative computer interpretations as determined from 419 patients, grouped under the panel 12 lead electrocardiogram interpretation

\begin{tabular}{lrrr}
\hline Panel interpretation & Totals & \multicolumn{2}{c}{$\begin{array}{l}\text { Computer } \\
\text { Interpretation }\end{array}$} \\
\cline { 2 - 4 } & & \multicolumn{2}{c}{ I2 lead 3 lead } \\
\hline Atrial abnormalities & 23 & 14 & 13 \\
Conduction defects & 29 & 4 & 13 \\
Left ventricular hypertrophy & 88 & 8 & 10 \\
Right ventricular hypertrophy & 25 & 5 & 7 \\
Myocardial infarction & 95 & 8 & 6 \\
Myocardial ischaemia & 128 & 14 & 10 \\
Miscellaneous & 31 & 5 & 6 \\
\hline \multicolumn{1}{c}{. } & 419 & 58 & 65 \\
\hline
\end{tabular}

tant false negative findings. Care has to be exercised in the analysis of these results-for example, in all 14 cases of false negative myocardial infarction from either system the abnormality was apparent on inspection. This implies that the computer had incorrectly determined the QRS onset and so had not accurately measured a $Q$ wave duration or a $0.03 \mathrm{sec}$ QRS vector angle. On the other hand, in a few examples of 12 lead false negative left ventricular hypertrophy it may have been that the computer had more correctly measured the I $\mathrm{mV}$ calibration signal than the panel. However, the 3 lead false negative left ventricular hypertrophy interpretations appeared valid on inspection - that is, there was no computer error, the 3 lead electrocardiogram being normal. When compared with the findings of Allenstein and Mori (1960), false positive 12 lead interpretations of left ventricular hypertrophy in 53 per cent of a sample of 32 normal hearts at necropsy, it is possible that the 3 lead electrocardiogram gave the more accurate interpretation by failing to report left ventricular hypertrophy. This point will be supported by further results described below.

Normal 12 lead electrocardiogram, abnormal 3 lead electrocardiogram A further study was made on those patients with a normal 12 lead but an abnormal 3 lead electrocardiogram and vice versa. Some details have previously been presented (Macfarlane, Lorimer, and Lawrie, 1969).

There were 213 patients with a normal 12 lead electrocardiogram, none of whom were used in the derivation of normal ranges of 3 lead parameters because of an abnormal cardiovascular system, or because cardiovascular abnormalities might be present but undetected - for example, patients with renal 
disease or because of a heart rate outside the range 60-100 per minute. After allowances were made for minor differences between 3 lead and 12 lead electrocardiogram computer interpretations (and excluding a few frank technical errors which caused false positive interpretations) there remained 263 lead electrocardiograms (12\%) which differed significantly from the 12 lead interpretation of normal. In 20 of these 26 patients $(77 \%$ ) the abnormal 3 lead electrocardiograms were consistent with the clinical findings (Table 5).

Normal 3 lead electrocardiogram, abnormal 12 lead electrocardiogram In the opposite situation there were 245 patients with a normal 3 lead electrocardiogram which was not used in the derivation of normal 3 lead parameters. There were 43 of these patients with abnormal 12 lead electrocardiograms, after due allowance was made for minor differences between 3 and 12 lead interpretations as in the previous group. In this case, however, only $15(35 \%)$ of the abnormal i2 lead electrocardiograms were consistent with the clinical findings (Table 6).

Comparison with respect to clinical classification Most patients who were clinically normal have already been reported in the section on false positive findings. A comparison of interpretations with respect to the remaining major clinical classifications will be described below. The question of computer error has not been considered in order to provide a direct comparison of lead systems. Thus if only one lead system computer interpretation reported an abnormality which was apparent on both types of electrocardiogram on inspection, this was not regarded as one lead system being superior to the other. Only true differences between corresponding interpretations, on the basis of the chosen criteria, were considered. These are shown in Table 7. The significant points are summarized below.

\section{Myocardial infarction}

There were II 4 patients in this group. Both lead systems diagnosed myocardial infarction in $86(75 \%)$ patients. The 12 lead electrocardiogram alone detected myocardial infarction in one patient, whereas the 3 lead alone detected inferior myocardial infarction in eight $(7 \%)$ patients. The occurrence of the latter was due either to the presence of small $R$ waves or the lack of $2 Q$ waves of suitable amplitude or duration in the inferior leads of the 12 lead electrocardiogram. The presence of a small $R$ wave in lead $Y$ of the 3 lead
TABLE 5 Relation between abnormal

3 lead electrocardiograms and the clinical

classification of the corresponding patients with

a normal I2 lead electrocardiogram

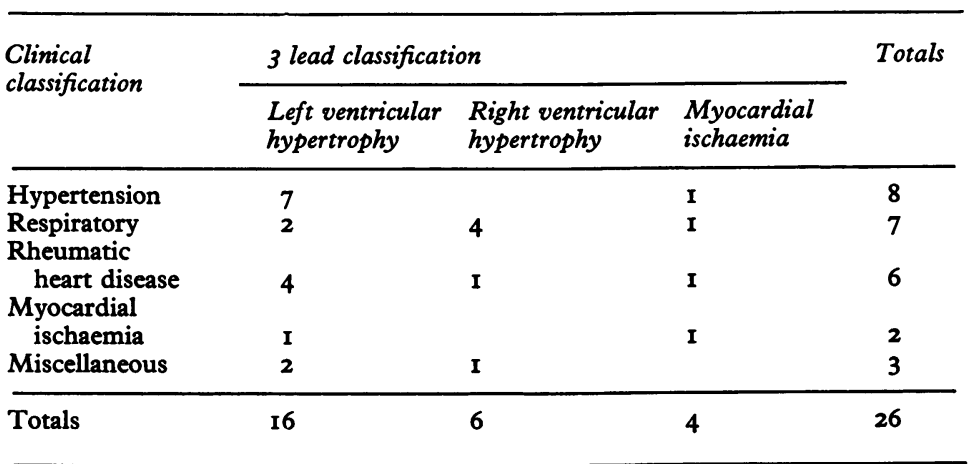

TABLE 6 Relation between abnormal I 2

lead electrocardiograms and the clinical

classification of the corresponding patients

with a normal 3 lead electrocardiogram

\begin{tabular}{|c|c|c|c|c|}
\hline \multirow{2}{*}{$\begin{array}{l}\text { Clinical } \\
\text { classification }\end{array}$} & \multicolumn{3}{|c|}{12 lead classification } & \multirow[t]{2}{*}{ Totals } \\
\hline & $\begin{array}{l}\text { Left ventricular } \\
\text { hypertrophy }\end{array}$ & $\begin{array}{l}\text { Right ventricular } \\
\text { hypertrophy }\end{array}$ & $\begin{array}{l}\text { Myocardial } \\
\text { ischaemia }\end{array}$ & \\
\hline Hypertension & 7 & & & 7 \\
\hline Respiratory & & I & & I \\
\hline \multicolumn{5}{|l|}{ Rheumatic } \\
\hline heart disease & 2 & & $\mathbf{I}$ & 3 \\
\hline Neurological & 5 & & 2 & 7 \\
\hline Miscellaneous & 6 & 2 & $\mathbf{I}$ & 9 \\
\hline Normal & 2 & 4 & 2 & 8 \\
\hline \multirow{3}{*}{$\begin{array}{l}\text { Endocrine } \\
\text { Myocardial } \\
\text { ischaemia }\end{array}$} & 4 & & I & 5 \\
\hline & & & & \\
\hline & & $\mathbf{I}$ & 2 & 3 \\
\hline Totals & 26 & 8 & 9 & 43 \\
\hline
\end{tabular}

electrocardiogram did not preclude the diagnosis of inferior myocardial infarction which was made on the basis of the orientation of the $0.03 \mathrm{sec}$. $Q R S$ vector and the $R / Q$ ratio in lead $\mathrm{Y}$. The diagnosis of ventricular hypertrophy due to an associated hypertension was made by one or other lead system in a few instances in almost equal proportions. There were no false positive findings in this group.

\section{Myocardial ischaemia}

There were 62 patients in this group. Both lead systems detected ST-T abnormalities of myocardial ischaemia in $22(35 \%)$ patients. The 3 lead electrocardiogram alone detected myocardial ischaemia in one patient and left ventricular hypertrophy in five $(8 \%)$ patients, 
TABLE 7 Differences between 3 lead (vectorcardiogram) and I2 lead (electrocardiogram) system interpretations. Each figure represents number of instances that a particular abnormality was detected by one lead system only. Figures do not include discrepancies due to computer error

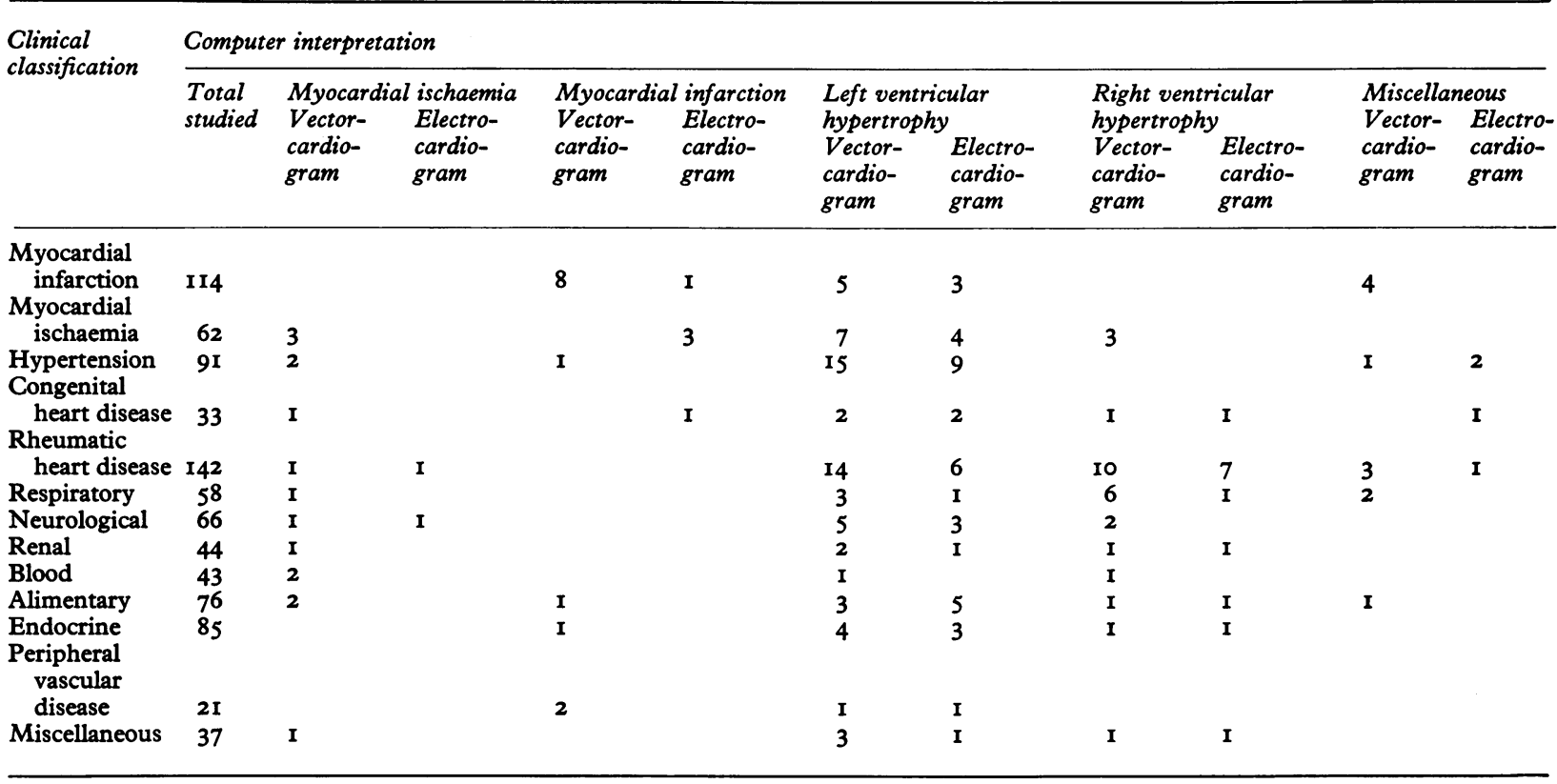

three of whom had systemic hypertension; the other two had left ventricular enlargement radiologically. The 12 lead electrocardiogram, while consistent with myocardial ischaemia, also suggested anteroseptal myocardial infarction in 3 patients, on the basis of low $R$ waves $(\mathrm{R}<0 \cdot \mathrm{IO} \mathrm{mV})$ or unusual small $\mathrm{Q}$ waves in leads $\mathrm{V}_{2}-\mathrm{V}_{4}$. The remaining four $(6 \%)$ I2 lead and seven (II\%) 3 lead electrocardiograms were regarded as false positive findings.

\section{Systemic hypertension}

There were 9r patients in this group. A few patients with systemic hypertension were included in other clinical categories because of their clinical presentation (for example, acute myocardial infarction). Left ventricular hypertrophy was detected by both lead systems in $23(25 \%)$ patients. In addition, 9 (I I \%) patients had evidence of left ventricular hypertrophy on the 12 lead electrocardiogram only and 15 (16\%) had evidence of left ventricular hypertrophy on the 3 lead electrocardiogram only. These findings were regarded as clinically expected, as were the other abnormalities detected by one or other lead system only. There were no false positive findings in this group.

\section{Congenital heart disease}

There were 33 patients in this group, and in general there was good agreement between interpretations of each lead system. The 3 lead electrocardiogram report of left ventricular hypertrophy was clinically expected in one patient with coarctation of the aorta, but the remaining discrepancies between lead system interpretations as listed in Table 7 were regarded as false positive findings.

\section{Rheumatic heart disease}

In this group of 142 patients there was agreement as regards left, right, or combined ventricular hypertrophy in $42(30 \%)$ patients. There were different interpretations in $43(30 \%)$ other patients. The 3 lead electrocardiogram alone in $13(9 \%)$ patients and the I2 lead alone in $3(2 \%)$ patients was reported as left ventricular hypertrophy, an interpretation which was consistent with the clinical findings of aortic or mitral valve disease or of both. The 3 lead electrocardiogram alone in $9(6 \%)$ patients, and the 12 lead alone in 6 $(4 \%)$ detected right ventricular hypertrophy where again the interpretation was consistent with the clinical diagnosis of predominant mitral stenosis. The remaining two $(\mathrm{r} \cdot 4 \%) 3$ lead and four (3\%) I2 lead electrocardiogram interpretations of ventricular hypertrophy were regarded as false positive. The other discrepancies between lead systems were consistent with the clinical findings in each instance. 


\section{Respiratory}

There were 58 patients in this group. Both lead systems detected right ventricular hypertrophy in only two $(3.5 \%)$ patients. The 3 lead electrocardiogram alone detected right ventricular hypertrophy in $6(10 \%)$ patients and the 12 lead alone did likewise in one patient only. These interpretations were consistent with the clinical findings, but the three $(5 \%) 3$ lead and one 12 lead cardiogram interpretations of left ventricular hypertrophy were regarded as false positive. The remaining three discrepancies were consistent with the clinical findings.

Neurological, renal, alimentary, endocrine, peripheral vascular disease, blood, miscellaneous There was a total of 372 subjects in these remaining groups, into which patients were placed on account of their presenting clinical features. It is realized that the different electrocardiographic abnormalities (if any) encountered in each group are not mutually exclusive and the classifications were for convenience to assist the derivation of normal ranges. The 3 lead electrocardiogram alone reported left ventricular hypertrophy in eight $(2 \%)$ patients, of whom seven had systemic hypertension, the other having Friedreich's ataxia, while the 12 lead electrocardiogram alone reported left ventricular hypertrophy in two patients. These interpretations were regarded as consistent with the clinical findings. There were $4(\mathrm{r} \%)$ patients with 3 lead evidence of ischaemic abnormalities which were clinically expected. In addition there were two 3 lead electrocardiogram interpretations which were consistent with inferior myocardial infarction, whereas the corresponding 12 lead electrocardiograms showed $Q$ waves of doubtful diagnostic significance. The other discrepancies, $26(7 \%) 3$ lead and I7 (5\%) I2 lead electrocardiogram, were regarded as false positive.

\section{Discussion}

Any large study of electrocardiograms including patients of all ages suffers from the limitation of lack of correlation between electrocardiographic abnormalities detected and the underlying pathology of the heart. Nevertheless it is necessary to define a border between normal and abnormal ranges of electrocardiographic parameters. For a diagnostic guide line a semi-arbitrary value has to be chosen. In this study the values incorporated in the newly developed 3 lead diagnostic criteria have proved effective throughout the spectrum of abnormalities, even though no necropsy correlations were used in their derivation. In our opinion the inclusion of such data would not materially have altered the findings for the following reasons. Firstly, the choice of diagnostic criteria was weighted heavily by the findings from the normal group of subjects - for example, the 96 per cent upper limit of normal for the maximum $Q R S$ vector amplitude was $2.31 \mathrm{mV}$ and that chosen for the diagnosis of left ventricular hypertrophy (in the presence of normal T waves) was $2.50 \mathrm{mV}$; this choice was made irrespective of the 96 per cent limits of the 3 lead parameter ranges in patients whose 12 lead electrocardiogram showed left ventricular hypertrophy. The same was true for other parameters used in this context - for example, $S_{z}, R_{x}$, and the angle in the transverse plane of the projection of the maximum $Q R S$ vector. On this basis of criteria formulation alone the 3 lead electrocardiogram allowed a greater differentiation of normal from abnormal patterns than did the 12 lead. Secondly, it is certain that in any study comparing normal hearts with those showing left ventricular hypertrophy (as determined at necropsy) there would be an overlap of ranges of 3 or 12 lead parameters from each group. The same problem of choice of normal values would then still arise.

In the estimation of false positive and negative computer interpretations the incidence of conduction defects has not been presented. For complete bundle-branch block both false positive and negative findings are due solely to technical or programming faults, which occurred on a few occasions. The problem, however, was complicated by the criterion used in the diagnosis of incomplete right bundle-branch block on the 12 lead electrocardiogram - namely, an $R^{\prime}{ }_{v 1}$ of any amplitude. In retrospect this was an oversimplification which presents a biased view of the occasional failure of the 3 lead electrocardiogram to detect incomplete right bundle-branch block on the basis of the above criterion. Such data have not been presented for this reason.

A comparison with the results of other workers can best be made by considering the incidence of false positive and negative computer interpretations of myocardial infarction with regard to cardiologists' interpretations of 12 lead electrocardiograms. This avoids the possibility (as for left ventricular hypertrophy) that for either lead system the computer may have given the correct interpretation of the electrocardiogram wave pattern, which was however regarded as false positive or negative in view of the clinical findings. Thus the actual computing technique error 
can be compared. In a study of 2060 I 2 lead electrocardiograms, Pordy et al. (I968) reported a false positive incidence of $28(\mathrm{I} \cdot 35 \%)$ interpretations of myocardial infarction. These compare with 17 (I.55\%) 3 lead and 25 $(2.28 \%)$ I2 lead electrocardiograms obtained from the ro93 subjects used in this study. With regard to false negative interpretations of myocardial infarction the figures are 9.7 per cent for Pordy et al. and 6.3 per cent (3 lead) and 8.4 per cent (12 lead) for this study (see Table 4).

While the analysis of arrhythmias was not undertaken, this in no way detracts from the comparison of lead systems. There is a belief that the 3 lead electrocardiogram cannot be used for arrhythmia analysis. This is incorrect, since the 3 lead can be displayed both in the conventional scalar form as well as a vectorcardiogram (Fig. 2). Analysis of arrhythmias could be made from the former but not from the latter.

On a number of occasions only one of the 3 or 12 lead systems detected a significant abnormality. While this was slightly to the advantage of the 3 lead system, there was no conspicuous difference between lead systems. It is clear, however, that the optimal lead system has not yet been devised. It seems likely that continual revision of 3 lead diagnostic criteria could lead to improvement in diagnostic accuracy, whereas this does not seem probable with the 12 lead. The reason for this is the ability of the 3 lead system to detect abnormally orientated cardiac electromotive forces by preserving the time relationship between lead potentials. Though new techniques such as multiple dipole electrocardiography (Holt et al., 1969) are promising, these require considerably more time both for recording and analysis and are cumbersome for female patients. In addition, since the multiple dipoles are constrained positive, analysis of the ST-T segment cannot be made with present theoretical methods. There is no doubt that 3 or I2 lead electrocardiography will continue in use for many years hence.

\section{Conclusions}

The results of this study show that the 3 lead electrocardiogram is clinically as useful as the

12 lead when interpretation is made by automated methods. No appreciable difference

was found between lead systems with respect to false positive or negative computer interpretations. In patients where one or other lead system showed a normal pattern the 3 lead showed greater correlation with the clinical findings and had fewer false positive findings. In the comparison by clinical classification the 3 lead cardiogram always detected more clinically expected abnormalities than did the 12 lead.

Computer assistance enhances interpretation of the 3 lead electrocardiogram, which requires about one-third of the computer time for analysis as compared to the 12 lead. This has a clear advantage for routine purposes, and adds weight to the overall conclusion that the use of 3 orthogonal lead electrocardiography is the method of choice for computer interpretation of electrocardiograms.

\section{References}

Allenstein, B. J., and Mori, H. (1960). Evaluation of electrocardiographic diagnosis of ventricular hypertrophy based on autopsy comparison. Circulation, 2I, 401 .

Berson, A. S., and Pipberger, H. V. (1967). Electrode to skin impedance problems in ECG recordings. In Digest of the 7th International Conference on Medical and Biological Engineering, Stockholm, 1967, p. 415.

Bonner, R. E., and Schwetman, H. D. (I968). Computer diagnosis of electrocardiograms. II. A computer program for EKG measurements. Computers and Biomedical Research, I, 366.

Borun, E. R., Chapman, J. M., and Massey, F. J. (I966). Computer analysis of Frank lead electrocardiographic data recorded in an epidemiologic study. American fournal of Cardiology, 18, 664.

Brody, D. A., and Arzbaecher, R. C. (1964). A comparative analysis of several corrected vectorcardiographic leads. Circulation, 29, 533.

Caceres, C. A., Steinberg, C. A., Abraham, S., Carbery, W. J., McBride, J. M., Tolles, W. E., and Rikli, A. E. (1962). Computer extraction of electrocardiographic parameters. Circulation, 25, 356.

Frank, E. (1956). An accurate, clinically practical system for spatial vectorcardiography. Circulation, 13, 737.

Holt, J. H., Barnard, A. C. L., Lynn, M. S., and Svendsen, P. (1969). A study of the heart as a multiple dipole electrical source. I. Normal adult male subjects. Circulation, 40, 687.

Macfarlane, P. W. (1969). A modified axial lead system for orthogonal lead electrocardiography. Cardiovascular Research, 3, 510.

- (1971). ECG waveform identification by digital computer. Cardiovascular Research 5, I4I.

- Lorimer, A. R. L., and Lawrie, T. D. V. (1969). Computer interpretation of normal ECGs and corresponding VCGs. In Proceedings of the Xth International Colloquium on Vectorcardiography, Warsaw. In the press.

,-- and - (197 I). Normal ranges of modified axial lead system electrocardiogram parameters. British Heart fournal, 33, 258.

McFee, R., and Parungao, A. (196I). An orthogonal lead system for clinical electrocardiography. American Heart fournal, 62, 93.

New York Heart Association (1964). Diseases of the Heart and Blood Vessels: Nomenclature and Criteria for Diagnosis, 6th ed. J. and A. Churchill, London.

Pipberger, H. V., Bialek, S. M., Perloff, J. K., and Schnaper, H. W. (I96I). Correlation of clinical information in the standard I2-lead ECG and a corrected orthogonal 3-lead ECG. American Heart fournal, 6r, 34 . 
, and Stallmann, F. W. (1964). Computation of differential diagnosis in electrocardiography. Annals of the New York Academy of Sciences, 115, III5.

Pordy, L., Jaffe, H., Chesky, K., Friedberg, C. K., Fallowes, L., and Bonner, R. E. (1968). Computer diagnosis of electrocardiograms. IV. A computer program for contour analysis with clinical results of rhythm and contour interpretation. Computers and Biomedical Research, $\mathrm{I}, 408$.

Simonson, E. (196I). Differentiation Between Normal and Abnormal in Electrocardiography. C. V. Mosby, St. Louis.

, Tuna, N., Okamoto, N., and Toshima, H. (I966). Diagnostic accuracy of the vectorcardiogram and electrocardiogram. American fournal of Cardiology, 17, 829.

Smith, R. E., and Hyde, C. M. (1969). Computer analysis of the electrocardiogram in clinical prac- tice. In Electrical Activity of the Heart, p. 305. Ed. by G. W. Manning and S. P. Ahuja. Thomas, Springfield, Illinois.

Sokolow, M., and Lyon, T. P. (1949a). The ventricular complex in left ventricular hypertrophy as obtained by unipolar precordial and limb leads. American Heart fournal, 37, 16r.

- and - (r949b). The ventricular complex in right ventricular hypertrophy as obtained by unipolar precordial and limb leads. American Heart fournal, 38, 273.

Spach, M. S., Barr, R. C., Havstad, J. W., and Long, E. C. (1966). Skin-electrode impedance and its effect on recording cardiac potentials. Circulation, $34,649$.

Stallmann, F. W., and Pipberger, H. V. (196I). Automatic recognition of electrocardiographic waves by digital computer. Circulation Research, 9, 1138. 